\title{
NanoCrystal: A Web-Based Crystallographic Tool for the Construction of Nanoparticles Based on
}

\section{Their Crystal Habit}

Alexios Chatzigoulas ${ }^{1,2}$, Konstantina Karathanou $^{1,2, \dagger}$, Dimitris Dellis $^{3}$, Zoe Cournia ${ }^{1, *}$

${ }^{1}$ Biomedical Research Foundation, Academy of Athens, 4 Soranou Ephessiou, 11527 Athens, Greece.

${ }^{2}$ Information Technologies in Medicine and Biology, Department of Informatics and Telecommunications, National and Kapodistrian University of Athens, Ilisia, 15784, Athens.

${ }^{3}$ Greek Research and Technology Network, S.A., 7 Kifissias Ave, 11523 Athens.

*Corresponding Author

E-mail: zcournia@bioacademy.gr, Phone number: +30 2106597195

$\dagger$ Present Addresses

Department of Physics, Freie Universität Berlin, Arnimallee 14, 14195 Berlin, Germany 


\begin{abstract}
Modeling nanoparticles is an essential first step to assess their capacity in different uses such as in energy storage or drug delivery. However, creating an initial starting conformation for modeling and simulation is tedious because every crystalline material grows with a different crystal habit. In this application note, we describe Nano-Crystal, a novel web-based crystallographic tool, which creates nanoparticle models from any crystal structure guided by their preferred equilibrium shape under standard conditions according to the Wulff morphology (crystal habit). Users can upload a cif file, define the Miller indices and their corresponding minimum surface energies according to the Wulff construction of a particular crystal, and specify the size of the nanocrystal. As a result, the nanoparticle is constructed and visualized, and the coordinates of the atoms are output to the user. Nano-Crystal can be accessed and used at http://nanocrystal.vi-seem.eu/.
\end{abstract}




\section{INTRODUCTION}

Nanomaterials and nanomaterial-based technologies enable new applications across disciplines, including mechanical and electrical engineering, environmental cleanup, energy storage, fabrics, information and communication technologies, medicine, and several others ${ }^{1-}$

${ }^{4}$. A nanomaterial is defined as a material with any external dimension in the nanoscale or having internal structure or surface structure with dimensions ranging from $1 \mathrm{~nm}$ to $100 \mathrm{~nm}$. Nanoparticles have a larger surface area per weight than conventional materials; the dimensions of nanoparticles are so close to atomic dimensions that an unusually high fraction of the total atoms are is present on their surfaces, therefore a nanoparticle has a surface more populated than the bulk ${ }^{5}$. Nanoparticles possess unique physicochemical properties due to their high surface area and nanoscale size. Due to their potential to increase the material surface area, e.g. in highly catalytic gold nanoparticles, enhance anti-bacterial material properties, e.g. in anti-microbial polymer foil with silver-doped tricalcium phosphate nanoparticles, improve the hardness of a material, e.g., in nanocomposites, affect the electron band gap of materials, e.g., in solar cells, augment electric properties, e.g., in nanofilled coatings, alter magnetic properties, e.g., magnetic separation, enable drug delivery, e.g., in drug nanocarriers, these materials allow for a wide range of applications. ${ }^{6,7}$ The possibilities to design different nanomaterials are vast, and consequently, computational methods are now routinely being employed in order to predict and design novel nanoparticles before they are tested experimentally. For example, computational approaches can significantly aid our efforts in nanoparticle formulation selection by allowing visualization of the molecular motions that contribute to processes that take place in key steps in the nanomedicine pipeline, such as drug encapsulation and release ${ }^{8,9}$, nanoparticle targeting, delivery and uptake ${ }^{10-15}$, and nanoparticle effects on cells and tissues ${ }^{16-18}$. Computational methodologies for modeling nanoparticles can be classified depending on the timescale and length scale they apply to 
study; these methods vary from ab-initio quantum calculations, where the full electronic structure of the systems is modeled either statically or for short times, to classical microscopic (atomistic) simulations, mesoscopic coarse-grained techniques, and up to macroscopic approaches, where systems are modeled in the continuum ${ }^{19,20}$.

Nanoparticles of metals, chalcogenides, nitrides, and oxides are often crystalline, and these are referred to as nanocrystals. Nanocrystals are of particular interest for their mechanical, electrical, magnetic, optical, chemical and other properties, and recently they have been implicated in oil refinement, solar panels, drug delivery, biosensors, cosmetics, etc ${ }^{21-28}$. Curie and Gibbs were the first to show that the equilibrium shape of a crystal is that which minimizes the surface free energy integrated over the entire surface for a fixed volume produced in conditions of perfect thermodynamic equilibrium, vacuum and at $0 \mathrm{~K}$; this equilibrium shape is termed "crystal habit" 29 . Therefore, s-Slight variations in the growth rates of different crystal planes in a given crystal structure lead to different crystal habits, which dictate the characteristic external shape of a nanocrystal ${ }^{30}$. The equations that minimize the integrated surface free energy of the crystal defining the equilibrium crystal shape were first formulated by Wulff ${ }^{31}$, and thus the equilibrium crystal shape is also referred to as "Wulff construction" as well as crystal habit.

Accurate multi-scale modeling of crystal nanoparticles ${ }^{32-34}$ requires careful consideration of the crystal habit. However, crystallographic files only contain the crystallographic unit cell and not its crystal habit. Therefore, creating the initial starting conformation of a nanocrystal for modeling and simulation is tedious, because there is no easy way to construct the Wulff morphology from the crystallographic unit cell. Freeware software packages to construct the Wulff morphology are available (e.g., Vesta ${ }^{35,36}$, Mercury $^{37-40}$, WinXMorph ${ }^{41,42}$ ), but these must be downloaded and installed, and in many cases, additional libraries or software programs are required. We present here, for the first time, a web-based tool, which can 
produce nanoparticles for simulation from any crystalline material according to the GibbsWulff minimum surface energy, given their crystallographic unit cell and minimum free energy growing planes.

\section{METHODS}

Notation, Space and Point Groups in Crystallography. A crystal structure is the description of the ordered arrangement of atoms, ions, and molecules in a solid that repeats in symmetric patterns along the three-dimensional space. The smallest group of particles in a crystalline material that constitutes a repeating pattern is called the unit cell of the structure (Figure S1). The crystal structure is defined as a combination of the crystal lattice with the motif (or else basis). A crystal lattice is a lattice with points that are the connection points between the unit cells, and the motif consists of the arrangement of the building blocks (atoms, molecules) of a unit cell. If we replace the lattice points with the motif it will generate a structure that is referred to as a crystal, which is the periodic translational arrangement of atoms in space (Figure S2). The crystal structures of all crystals can be classified in into seven crystal systems, according to the symmetry of the unit cells (Figure S3) and in into four centering types, which show the position of the atoms or molecules in the unit cell (Figure S4). The combination of the seven crystal systems with the centering types produces the 14 Bravais lattices that are the distinct lattice types, which when repeated can fill the whole space (Figure S5). A crystal system is also defined by a point group, which is a group of symmetry operations, like rotations or reflections (Figure S6) and by a space group, which is the combination of the 14 Bravais lattices with the point groups (Figure S7-S9) ${ }^{43}$.

To describe the crystal planes in the Bravais lattices, the Miller indices notation system is used $^{44}$. The orientation of a surface or a crystal plane may be defined by considering how the 
plane, or any parallel plane, intersects the main crystallographic axes of the solid. The application of a set of rules leads to the assignment of the Miller indices, which are determined by three integers $\mathrm{h}, \mathrm{k}$, and $\mathrm{l}(\mathrm{hkl})$ that quantify the intercepts and thus may be used to uniquely identify a family of lattice planes or surfaces (Figure S10). For more information on crystallography theory, please refer to the SI.

Equilibrium Crystal Shape. Gibbs proposed that a droplet or crystal will arrange itself such that its surface Gibbs free energy is minimized by assuming a shape of low minimum surface energy $^{29}$. Wulff made use of Gibbs theory and created a methodology to determine the equilibrium shape of a crystal based on its preferred growing planes (crystal habit) and minimum surface energies of fixed volume produced in conditions of perfect thermodynamic equilibrium, vacuum, and at $0 \mathrm{~K}$. He also showed that the distance $\mathrm{d}$ of a crystal plane from the origin is proportional to its minimum surface energy $\gamma^{45}$ :

$\mathrm{d}_{\mathrm{hkl}}=\lambda * \gamma_{\mathrm{hkl}}, \lambda=\mathrm{constant} \quad(1)$, where $d_{h k l}$ is the distance $d$ of the Miller plane hkl from the origin, and $\gamma_{\mathrm{hkl}}$ is its minimum surface energy. Equation 1 denotes that lower surface energy leads to a larger plane area closer to the origin, because when the surface energy $\gamma_{\mathrm{hkl}}$ is reduced, the distance of that surface from the origin $d_{\mathrm{hkl}}$ is also reduced, and as a result, that plane truncates the other planes at a greater extent (Figure 1). The Wulff construction, or crystal habit, results in a polyhedron with the following properties: a) The polyhedron depends only on ratios between surface energy values of the different planes, therefore they can be expressed in any unit provided that the units are the same for each input surface energy. b) The (hkl) planes with high surface tension (usually high-indexed ones) are less likely to appear in the equilibrium shape. c) High-index faces are usually hidden behind low-index ones, even if their $\gamma_{\mathrm{hkl}}$ is small. d) The extra energy associated with the formation of edges and vertices between two surfaces is neglected. e) The polyhedron bears the same symmetry (belonging to the same 
point group) as the crystal structure of the material. For the above-mentioned point d), Wulff construction consists only of planar facets, which intersect in sharp corners and sharp straight edges. In case the edge energies are not neglected, the crystal faces would grow to be rounded faces. For the appearance of rounded edges, the temperature should be over $0 \mathrm{~K}^{46}$. This effect will become visible once the temperature is raised in the simulation of the nanoparticle. In addition, the Wulff construction assumes an equilibrium shape according to the minimum surface Gibbs free energy in vacuum. However, the growth of these minimum free energy surfaces can be modulated by solution conditions, $\mathrm{pH}$, temperature changes, etc., resulting in a change on the crystal shape ${ }^{47}$. The growing planes of the crystal, which correspond to its minimum surface energies, can be used to extend the size and shape of the nanoparticle $^{48}$ (Figure 1). Below, we describe the methodology we use to generate the Wulff construction or crystal habit from the crystal structure unit cell of the nanoparticle material. For more information, see the SI.

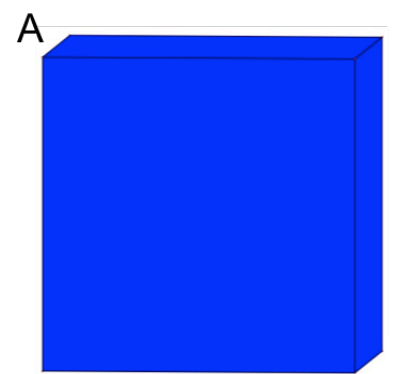

$$
\gamma_{\frac{111}{100}}=2
$$

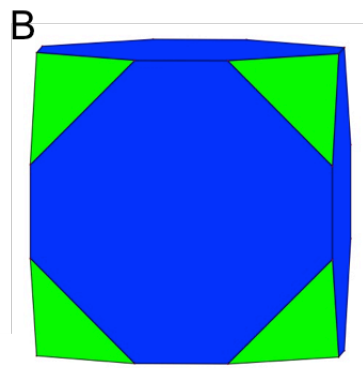

$$
\gamma_{\frac{111}{100}}=4 / 3
$$

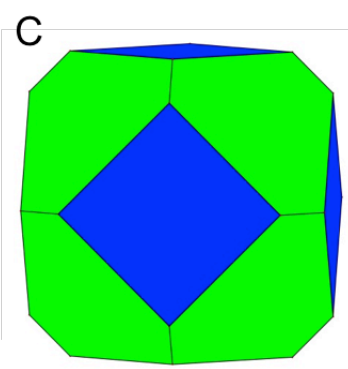

$$
\gamma_{\frac{111}{100}}=1
$$

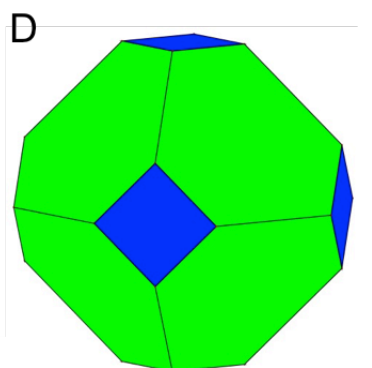

$\gamma_{\frac{111}{100}}=3 / 4$

Figure 1. Demonstration of the effect of the surface plane free energy ratios on the morphology of the crystal growing planes in a cubic crystal example. (A) The surface free energy of the $\{100\}$ plane family is dominating the morphology for the $\{111\} /\{100\}$ free energy ratio equal to 2 . (B) Upon decreasing the $\{111\} /\{100\}$ ratio to $4 / 3$, the $\{111\}$ Miller plane family becomes visible in the crystal morphology truncating the corners of the crystal shape. (C) Further decrease of the $\{111\} /\{100\}$ ratio to 1, results in a more evident 
appearance of the $\{111\}$ planes. (D) The $\{111\}$ Miller plane family dominates the crystal morphology, when the $\{111\} /\{100\}$ ratio is equal to $3 / 4$.

Minimum Energy Planes. First principles quantum mechanical calculations are important complementary tools to experimental techniques in characterizing surface properties of a material such as its surface energies ${ }^{49}$. The Wulff construction provides the crystal shape under equilibrium conditions, and in this construction the distance of a face from the origin is proportional to the surface energy of that face. As a result, the equilibrium shape is formed by the planes with the minimum free energy, and these can be predicted by ab-initio quantum mechanical calculations at the DFT, MP2, CCSD(T), etc. level of theory or by computational techniques such as the Donnay-Harker principles $^{50}$, the Hartman-Perdok method ${ }^{51-53}$, or the Ising model ${ }^{54}$, which can predict the crystal morphology. The minimum surface free energy planes and their associated energies are used in the present study as input from literature sources to construct the crystal habit.

Crystal Habit Calculation. By using the Wulff construction, our algorithm constructs the equilibrium shape of a crystalline nanoparticle via the following steps:

Step 1: First, the algorithm produces the symmetric Miller indices for each hkl triplet input, according to the point group of the crystal, which is taken from the crystallographic file. As a result, a list of all symmetric Miller indices is obtained. From each one of the hkl triplet in this list, three Cartesian coordinates are produced from the fractional coordinates as well as the unit cell parameters, which are enough to produce a plane (Figure S11). Subsequently, from the list of Miller indices, we obtain a list of the corresponding Miller planes. These planes are placed at a distance from the origin, which is proportional to their surface energy usingat a user-defined maximum nanoparticle radius. 
Step 2: The second step is the calculation of the polyhedron vertices. To do so, An exhaustive search is performed to find all the intersection points by taking all possible combinations of three Miller planes (Figure S12). The algorithm discards those intersection points that lie outside of at least one Miller plane (verified by using the plane equations); the rest of the intersection points form the set of the polyhedron vertices, V (Figure S13).

Step 3: The third step is the definition of the polyhedron faces. In principle, we note that each face is a polygon that lies on one of the planes, which it is defined by a subset of $\mathrm{V}$ and by a set $\mathrm{E}$ of ordered edges, i.e., line segments that connect vertices. For each Miller plane, all vertices that lie on it (satisfy the plane equation) are first defined. Thus, a polygon is created given these vertices using the Quickhull algorithm ${ }^{55}$. With the Quickhull algorithm, the convex hull of a set of points (in our case, the subset of the vertices) is defined. It uses the divide and conquer approach, and its average case complexity is considered to be $\mathrm{O}(\mathrm{n} * \log (\mathrm{n}))$, whereas in the worst case it takes $\mathrm{O}\left(\mathrm{n}^{2}\right)$, where $\mathrm{n}$ is the input size and in our case the number of vertices. In our implementation of the algorithm, the most distant points are connected with a (double) line segment, which initializes the set E. Then, in each of the iterative steps, the most distant point from any of the line segments in $\mathrm{E}$ is found; that line segment is replaced with two new ones, connecting the most distant point. The iterative steps of the algorithm stop when all points have been included in the convex hull (Figures S14S15).

In this way, for each Miller plane we obtain an ordering of the edges that form a closed chain. As a result, the faces of the equilibrium shape are obtained, and the equilibrium shape of the nanoparticle is constructed. Therefore, with the preferred growing planes of the crystal in Miller indices, their corresponding minimum surface energies (Wulff construction), and the desired maximum nanoparticle radius, the equilibrium shape (Wulff morphology) of any 
crystal of any space group, lattice parameters, and size, is constructed. The workflow for the construction of the Wulff morphology is presented in Figure S16.

Unit cell replication. Now that the crystal habit of the nanoparticle has been constructed, the next step is to fill that shape with the atoms of the material. The first step to fill the equilibrium shape with atoms is to calculate the symmetric unit cell from the asymmetric one. The asymmetric unit refers to the smallest possible occupation of space within the unit cell (Figure S17). The algorithm applies the symmetry operations and crystal parameters of the crystal space group from the crystallographic file to the atoms of the asymmetric unit cell. This procedure produces the symmetric unit cell and its atoms. The steps to build the symmetric unit cell from the asymmetric one are as follows:

Step 1: Apply the given symmetry operations (obtained from the crystallographic file) to the atoms of the asymmetric unit and remove duplicated atoms. As a result, a list of atoms is obtained with their Cartesian coordinates (Figure S18).

Step 2: Atoms that are outside the asymmetric unit cell should be moved inside. The asymmetric unit cell is a cube with lengths of $1 \AA$ with the origin being on the bottom left edge. If one of the Cartesian coordinates of the atom is below 0 or over 1 , we add or subtract 1 , respectively.

Step 3: Atoms that are on the unit cell faces should be copied to its parallel face.

Step 4: Atoms that are on an edge should be copied to all edges.

Step 5: Apply the lattice parameters on atomic coordinates of the asymmetric unit. The lattice parameters (unit cell lengths and angles) are not present in the asymmetric unit cell and as a result in the given atom coordinates in the cif file. So we have to apply them in order to get the atom coordinates of the symmetric unit cell. 
As a result of this methodology, we obtain the symmetric unit cell of the crystal (Figure S19).

The second step in order to fill the equilibrium shape with atoms is to replicate the symmetric unit cell along all three spatial directions until the equilibrium shape is filled. To do that is, The algorithm replicates the symmetric unit cell across all directions until it reaches the maximum length of $\mathrm{x}, \mathrm{y}$, and $\mathrm{z}$ of the equilibrium shape. As a result, a big cube or parallelepiped is built (Figure S20). Then the duplicated atoms and the atoms that are outside of the equilibrium shape (Figure S21) are removed. The final nanoparticle is constructed (Figure S22), as a result of these steps (Figure S23). In Figures 2 and 3 we demonstrate the workflow of the methodology.

Coordination polyhedra. Upon constructing the crystal habit of the nanoparticle, the user has the option to create the crystal habit by truncating the shape exactly at the crystal habit boundary or to include all atoms that form coordination polyhedra for atoms inside the equilibrium shape. To implement this feature we used the average single-bond covalent radii list from analysis of the Cambridge Structural Database, based on a statistical analysis of more than 228,000 experimental bond lengths ${ }^{56}$. Then, we compute the bond between atoms $\mathrm{A}$ and $\mathrm{B}$, from the summation of their covalent radii and a tolerance value of $0.2 \AA$ (see Supplementary Information for mode details). As a result, the coordination polyhedra on the boundary of the equilibrium shapes are kept intact (Figure S24).

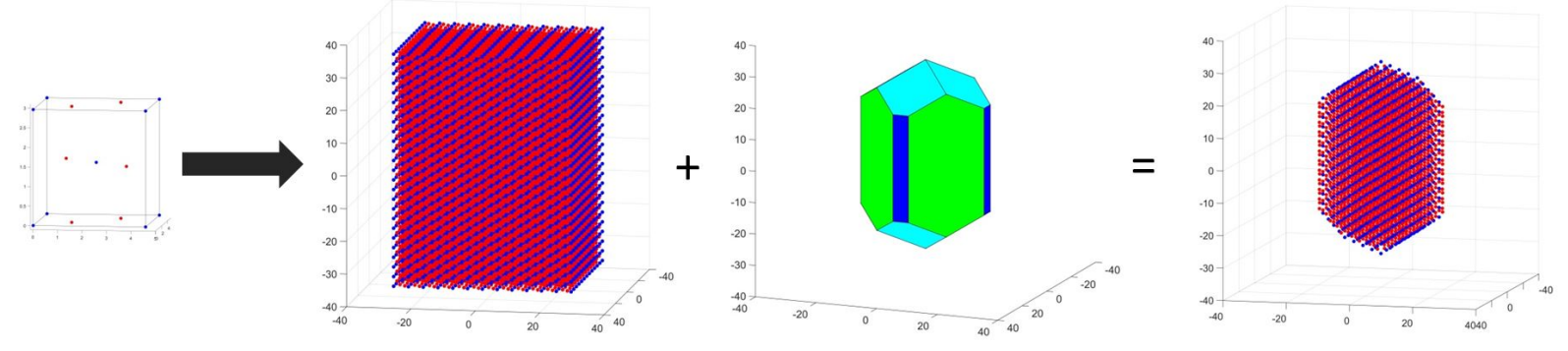

Figure 2. The visualization of the algorithm's methodology using Matlab visualizations. 
Validation. For validating our code, we have used the following approaches. First, we selected literature cases, where the crystal habit is constructed by using input from quantum mechanical calculations. Using this input we reproduced the crystal habit with our web server. Specifically, we retrieved quantum mechanical information for the cases of: (a) $\mathrm{Fe}_{3} \mathrm{O}_{4}$ (magnetite) nanoparticle, using data from Ref. ${ }^{57}$, (b) $\mathrm{TiO}_{2}$ (rutile) nanoparticle, using data from Ref. ${ }^{58}$, (c) $\mathrm{LiFePO}_{4}$ (olivine) nanoparticle using data from Ref. ${ }^{59}$. Second, the input from the above-mentioned crystal habit parameters was also input in $\mathrm{Vesta}^{35,36}$. We then checked whether the Vesta output is in agreement with the output of our algorithm. Third, we compared our results with experimental data. However, as experimental data do not provide surface energies, our comparison was limited to identifying the correct shape provided by the experiment. We have tested our web server against Ref.$^{60}$ and successfully reproduced the crystal habit of gold nanocrystals as retrieved by field emission scanning electron microscopy based on the experimentally provided Miller planes (refer to pages 34-35 in the SI and Figure S40).

\section{WEB SERVER IMPLEMENTATION}

Development. For the above-mentioned methodology and the web server, a new algorithm was programmed in both MATLAB and $\mathrm{C}++$, and no previously developed algorithms were re-implemented. $\mathrm{C}++$ runs in the backend in the current web server implementation, and the front-end is written in PHP. To visualize the nanoparticles after construction, the JSmol applet was added in to the website, which is a fully functional implementation of $\mathrm{Jmol}^{61,62}$ that does not require Java and runs in any modern (HTML5) web browser. A user manual and a test case for the construction of the $\mathrm{Fe}_{3} \mathrm{O}_{4}$ (magnetite) nanoparticle are included in the website. 


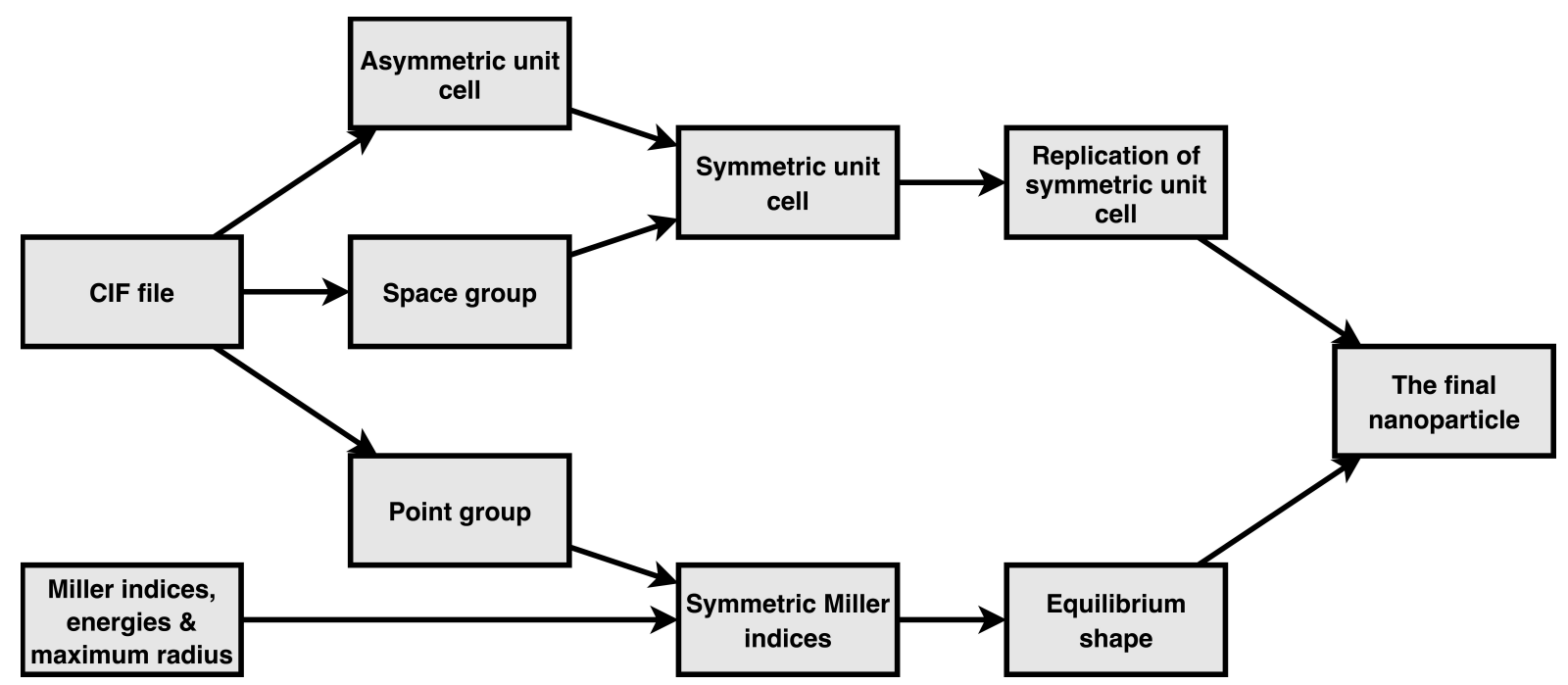

Figure 3. Workflow of the NanoCrystal tool.

User provided input. Crystal structures of materials are usually provided as .cif files ${ }^{63-67}$.

Therefore, the user inputs the cif file of the desired material in the web server, which contains all relevant crystal information such as crystal parameters, point group, space group, the atomic Cartesian coordinates of the asymmetric unit cell, etc. (Figure S25). Subsequently, the user inputs the Miller indices of the minimum energy growing planes and their corresponding surface energies (Figure S26-S28), which can be retrieved either from quantum mechanical calculations based on the Wulff construction. These energies can be expressed in any unit provided that the units are the same for each input surface energy because the Wulff construction results in a polyhedron that depends only on ratios between surface energy values and not on their absolute values. The user is also asked to choose whether to include all atoms that form coordination polyhedra for atoms inside the equilibrium shape. The final input is the maximum radius of the nanoparticle in $\AA$, which determines the size of the nanoparticle (Figure S28). After pressing the upload button, the web server produces the coordinates of the atoms in a .xyz file and a .pdb file and also visualizes the nanoparticle using the Jsmol plugin (Figure S30). 


\section{CASE STUDY}

The proposed workflow is demonstrated to construct the magnetite $\left(\mathrm{Fe}_{3} \mathrm{O}_{4}\right)$ nanoparticle. The first user-defined input is the cif file of the crystal. The magnetite crystallographic file can be downloaded from http://crystallography.net/cod/9010940.html ${ }^{68}$. Magnetite has an inverse spinel crystal structure based on a face-centered cubic array of oxide ions with magnetite $\mathrm{Fe}^{+2}$ occupying octahedral holes and $\mathrm{Fe}^{+3}$ equally distributed between octahedral and tetrahedral holes. The unit cell lengths are $a=b=c=8.3198 \AA$ and cell angles $\alpha=\beta=\gamma=90^{\circ}$. The magnetite cif file also contains the symmetry operations and the Cartesian coordinates of the atoms of the asymmetric unit. The Miller indices and minimum surface energies of magnetite are retrieved from a density factional theory (DFT) study ${ }^{57}$ (Table S1). The last user-defined input is the maximum radius of the NP in $\AA$ (for this case study we input $30 \AA$ ).

Using the Miller indices input and the point group of the crystal, the algorithm produces a total of 14 symmetric Miller planes and the equilibrium shape is produced (Figure 4A, Figure S31). Then the tool produces the symmetric unit cell and translates it across all directions until the above equilibrium shape is filled. As a result, the $\mathrm{Fe}_{3} \mathrm{O}_{4}$ nanoparticle is produced, and the coordinates of the atoms can be downloaded in a .pdb and a .xyz format (Figures S32-S33). The total number of the atoms is 11,222 . Figure $4 \mathrm{~A}$ presents the equilibrium crystal shape from the Wulff construction as generated by our algorithm and visualized in $\operatorname{Matlab}^{69}$, which is in agreement with the one from first principle calculations ${ }^{57}$, Figure 4B shows the final constructed $\mathrm{Fe}_{3} \mathrm{O}_{4}$ nanoparticle visualized using JSmol, and Figure 4C shows the final constructed $\mathrm{Fe}_{3} \mathrm{O}_{4}$ nanoparticle visualized using JSmol, including atoms that form coordination polyhedral with atoms inside the equilibrium shape (for more use cases see the SI, Figures S34-S40 and Tables S2-S3). 

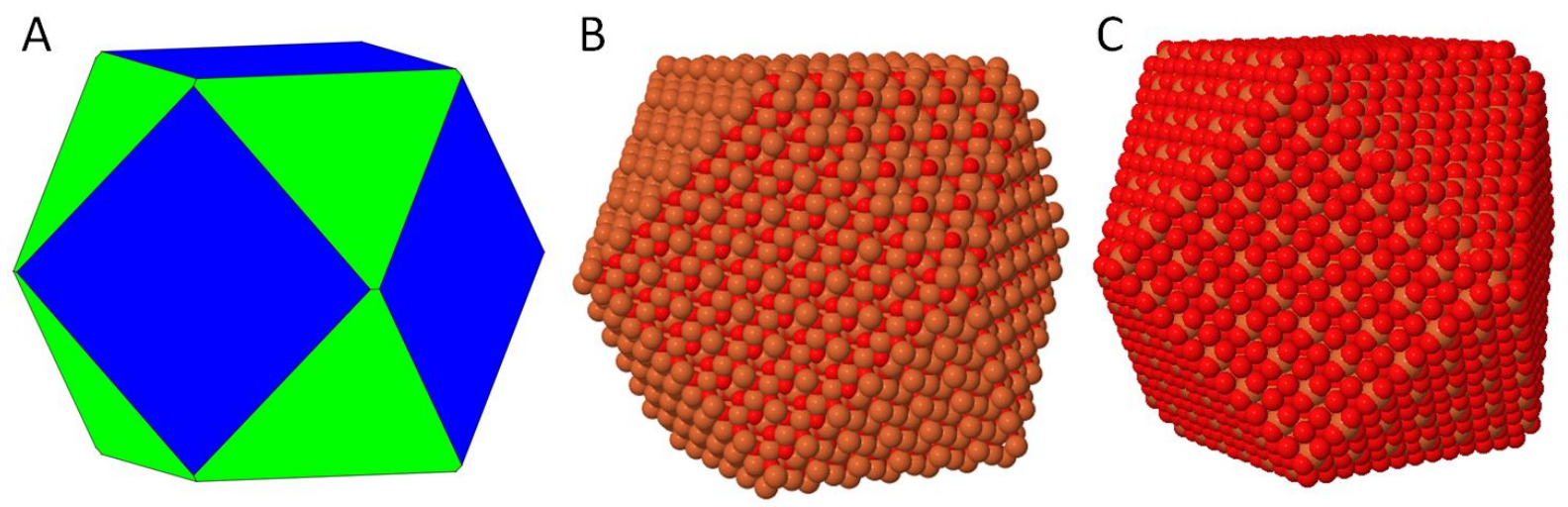

Figure 4. (A) The equilibrium shape of the $\mathrm{Fe}_{3} \mathrm{O}_{4}$ produced in Matlab. (B) The $\mathrm{Fe}_{3} \mathrm{O}_{4}$ nanoparticle visualized using JSmol. (C) The $\mathrm{Fe}_{3} \mathrm{O}_{4}$ nanoparticle visualized using JSmol, including atoms in coordination polyhedra.

\section{CONCLUSIONS}

In recent years, nanotechnology has attracted significant interest in many industrial applications. Building the initial configuration of a crystalline nanoparticle for simulation is a tedious task that requires specialized software to ensure that the equilibrium crystal shape of the material is taken into account. We present here, for the first time, a web-based tool, which can produce nanoparticles for simulation from any crystalline material according to the Gibbs-Wulff minimum surface energy, given their crystallographic unit cell and minimum free energy growing planes.

Our web server is a tool for scientists interested in efficiently retrieving atomic coordinates for crystal nanoparticles simply by entering the bulk structure and the surface energies of the relevant facets, and without the need for installing additional software. Other popular software for crystal structure visualization, exploration, and analysis such as Vesta ${ }^{35,36}$ and 
Mercury $^{37-40}$, provide additional functionalities; for example in Vesta the user may modify crystallographic parameters such as the lattice parameters, the point group, the space group, the atomic coordinates or model electron and nuclear densities, providing powder diffraction patterns etc., which are, however, beyond the scope of this work.

An algorithm was implemented that constructs the Wulff morphology for a any given crystal structure based on its preferred growing planes expressed in Miller indices and their corresponding surface energies (crystal habit) as well as a user-defined crystal size. Initially, the equilibrium crystal shape is created as a polyhedron, which is the intersection of multiple polyhedra and individual planes. The next step is to construct the symmetric unit cell from the asymmetric one and replicate it inside the previously constructed polyhedron. As a result, the final nanoparticle is constructed, and the coordinates of the atoms are written in .xyz and .pdb files, they can be downloaded. The nanoparticle can also be visualized within the website.

Future updates may include constructing more complex nanoparticle systems, such as constructing coated nanocrystals with user-defined ligands for biomedical simulations ${ }^{21}$ (Figure S41). Moreover, in case the Miller indices and their corresponding minimum surface energies are not known from literature sources, the Wulff construction may be automatically calculated by applying computational methods such as the Donnay-Harker principles ${ }^{50}$, the Hartman-Perdok method ${ }^{51-53}$ or the Ising model $^{54}$ (for more information about these methods refer to the SI and Figure S42). 


\section{ASSOCIATED CONTENT}

Supporting Information.

Additional methodological details and supporting figures explaining the methodology are presented in PDF format. A supporting video showcasing the web server use is provided in mp4 format. Four different use cases for nanocrystals $\mathrm{F}_{3} \mathrm{O}_{4}, \mathrm{LiFeO}_{4}, \mathrm{TiO}_{2}$, and $\mathrm{Au}$ are also provided with their accompanying cif files and Miller indices and minimum surfaces planes. The Supporting Information is available free of charge on the ACS Publications website.

\section{AUTHOR INFORMATION}

*Corresponding Author

E-mail: zcournia@bioacademy.gr

Phone number: +30 2106597195

Present Addresses

$†$ Department of Physics, Freie Universität Berlin, Arnimallee 14, 14195 Berlin, Germany

\section{AUTHOR CONTRIBUTIONS}

The manuscript was written through contributions of all authors. All authors have given approval to the final version of the manuscript.

\section{FUNDING SOURCES}

We acknowledge computational time granted from the Greek Research \& Technology Network (GRNET) in the National HPC facility - ARIS under project ID pr001026-MAGNANO-MEM. AC and ZC were co-funded by the European Commission under the H2020 
Research Infrastructures Contract No. 675121 (project VI-SEEM). The web server is hosted using VI-SEEM resources.

\section{CONFLICT OF INTEREST STATEMENT}

The authors declare no competing financial interest.

\section{ACKNOWLEDGMENT}

AC thanks Dr. Koichi Momma, the author of Vesta for his precious help during the development of this tool and for providing his input on implementing crystallography concepts.

\section{REFERENCES}

1. Lahann, J., Environmental Nanotechnology: Nanomaterials Clean Up. Nat. Nanotechol. 2008, 3, 320.

2. Von Maltzahn, G.; Park, J.-H.; Lin, K. Y.; Singh, N.; Schwöppe, C.; Mesters, R.; Berdel, W. E.; Ruoslahti, E.; Sailor, M. J.; Bhatia, S. N., Nanoparticles That Communicate in Vivo to Amplify Tumour Targeting. Nat. Mater. 2011, 10, 545.

3. Wu, X. L.; Jiang, L. Y.; Cao, F. F.; Guo, Y. G.; Wan, L. J., LiFePO4 Nanoparticles Embedded in a Nanoporous Carbon Matrix: Superior Cathode Material for Electrochemical Energy-Storage Devices. Adv. Mater. 2009, 21, 2710-2714.

4. Durán, N.; Marcato, P. D.; De Souza, G. I.; Alves, O. L.; Esposito, E., Antibacterial Effect of Silver Nanoparticles Produced by Fungal Process on Textile Fabrics and Their Effluent Treatment. J. Biomed. Nanotechnol. 2007, 3, 203-208.

5. Rao, C.; Thomas, P.; Kulkarni, G. Basics of Nanocrystals. In Nanocrystals: Synthesis, Properties and Applications, Rao, C.; Thomas, P.; Kulkarni, G., Eds.; Springer-Verlag Berlin Heidelberg: Heidelberg, 2007, pp 1-23.

6. Nagarajan, R. Nanoparticles: Building Blocks for Nanotechnology. In; ACS Publications: 2008.

7. Stark, W. J.; Stoessel, P. R.; Wohlleben, W.; Hafner, A., Industrial Applications of Nanoparticles. Chem. Soc. Rev. 2015, 44, 5793-5805.

8. Hossain, S. S.; Hossainy, S. F. A.; Bazilevs, Y.; Calo, V. M.; Hughes, T. J. R., Mathematical Modeling of Coupled Drug and Drug-Encapsulated Nanoparticle Transport in Patient-Specific Coronary Artery Walls. Comput. Mech. 2012, 49, 213-242. 
9. Huynh, L.; Neale, C.; Pomès, R.; Allen, C., Computational Approaches to the Rational Design of Nanoemulsions, Polymeric Micelles, and Dendrimers for Drug Delivery. Nanomed. Nanotechnol. Biol. Med. 2012, 8, 20-36.

10. Liu, Y.; Shah, S.; Tan, J., Computational Modeling of Nanoparticle Targeted Drug Delivery. Rev. Nanosci. Nanotechnol. 2012, 1, 66-83.

11. Ece Gamsiz, D.; Shah, L. K.; Devalapally, H.; Amiji, M. M.; Carrier, R. L., A Model Predicting Delivery of Saquinavir in Nanoparticles to Human Monocyte/Macrophage (Mo/Mac) Cells. Biotechnol. Bioeng. 2008, 101, 1072-1082.

12. Goodman, T. T.; Chen, J.; Matveev, K.; Pun, S. H., Spatio-Temporal Modeling of Nanoparticle Delivery to Multicellular Tumor Spheroids. Biotechnol. Bioeng. 2008, 101, 388-399.

13. Kim, B.; Han, G.; Toley, B. J.; Kim, C.-k.; Rotello, V. M.; Forbes, N. S., Tuning Payload Delivery in Tumour Cylindroids Using Gold Nanoparticles. Nat. Nanotechol. 2010, $5,465-472$.

14. Shah, S.; Liu, Y.; Hu, W.; Gao, J., Modeling Particle Shape-Dependent Dynamics in Nanomedicine. J. Nanosci. Nanotechno. 2011, 11, 919-928.

15. Xu, X.; Li, R.; Ma, M.; Wang, X.; Wang, Y.; Zou, H., Multidrug Resistance Protein P-Glycoprotein Does Not Recognize Nanoparticle C60: Experiment and Modeling. Soft Matter 2012, 8, 2915-2923.

16. Fetterly, G. J.; Grasela, T. H.; Sherman, J. W.; Dul, J. L.; Grahn, A.; Lecomte, D.; Fiedler-Kelly, J.; Damjanov, N.; Fishman, M.; Kane, M. P.,

Pharmacokinetic/Pharmacodynamic Modeling and Simulation of Neutropenia During Phase I Development of Liposome-Entrapped Paclitaxel. Clin. Cancer Res. 2008, 14, 5856-5863.

17. Lin, H.-Y.; Landersdorfer, C. B.; London, D.; Meng, R.; Lim, C.-U.; Lin, C.; Lin, S.; Tang, H.-Y.; Brown, D.; Van Scoy, B., Pharmacodynamic Modeling of Anti-Cancer Activity of Tetraiodothyroacetic Acid in a Perfused Cell Culture System. PLoS Comp. Biol. 2011, 7, e1001073.

18. Kaddi, C. D.; Phan, J. H.; Wang, M. D., Computational Nanomedicine: Modeling of Nanoparticle-Mediated Hyperthermal Cancer Therapy. Nanomedicine 2013, 8, 1323-1333.

19. Johnston, K.; Harmandaris, V., Hierarchical Simulations of Hybrid Polymer-Solid Materials. Soft Matter 2013, 9, 6696-6710.

20. Bradley, R.; Radhakrishnan, R., Coarse-Grained Models for Protein-Cell Membrane Interactions. Polymers 2013, 5, 890-936.

21. Patitsa, M.; Karathanou, K.; Kanaki, Z.; Tzioga, L.; Pippa, N.; Demetzos, C.; Verganelakis, D. A.; Cournia, Z.; Klinakis, A., Magnetic Nanoparticles Coated with Polyarabic Acid Demonstrate Enhanced Drug Delivery and Imaging Properties for Cancer Theranostic Applications. Sci.Rep. 2017, 7.

22. Angelikopoulos, P.; Sarkisov, L.; Cournia, Z.; Gkeka, P., Self-Assembly of Anionic, Ligand-Coated Nanoparticles in Lipid Membranes. Nanoscale 2017, 9, 1040-1048.

23. Gkeka, P.; Angelikopoulos, P.; Sarkisov, L.; Cournia, Z., Membrane Partitioning of Anionic, Ligand-Coated Nanoparticles Is Accompanied by Ligand Snorkeling, Local Disordering, and Cholesterol Depletion. PLoS Comp. Biol. 2014, 10, e1003917.

24. Beek, W. J.; Wienk, M. M.; Janssen, R. A., Efficient Hybrid Solar Cells from Zinc Oxide Nanoparticles and a Conjugated Polymer. Adv. Mater. 2004, 16, 1009-1013.

25. Crossley, S.; Faria, J.; Shen, M.; Resasco, D. E., Solid Nanoparticles That Catalyze Biofuel Upgrade Reactions at the Water/Oil Interface. Science 2010, 327, 68-72.

26. Yu, Y.; Sun, Q.; Zhou, T.; Zhu, M.; Jin, L.; Shi, G., On-Line Microdialysis System with Poly (Amidoamine)-Encapsulated Pt Nanoparticles Biosensor for Glutamate Sensing in Vivo. Bioelectrochemistry 2011, 81, 53-57. 
27. Puglia, C.; Bonina, F., Lipid Nanoparticles as Novel Delivery Systems for Cosmetics and Dermal Pharmaceuticals. Expert Op. Drug Del. 2012, 9, 429-441.

28. Kokura, S.; Handa, O.; Takagi, T.; Ishikawa, T.; Naito, Y.; Yoshikawa, T., Silver Nanoparticles as a Safe Preservative for Use in Cosmetics. Nanomed. Nanotechnol. Biol. Med. 2010, 6, 570-574.

29. Gibbs, J. W., On the Equilibrium of Heterogeneous Substances. Am. J. Sci. 1878, 441-458.

30. Prywer, J., Theoretical Analysis of Changes in Habit of Growing Crystals in Response to Variable Growth Rates of Individual Faces. J. Cryst. Growth 1999, 197, 271285.

31. Wulff, G., Some Theorems Concerning the Growth and Dissolution Rates of Crystals. Ger.), Z. Kristallogr 1901, 34, 449-530.

32. Hadjisavvas, G.; Remediakis, I. N.; Kelires, P. C., Shape and Faceting of Si Nanocrystals Embedded in A-SiO2: A Monte Carlo Study. Phys. Rev. B 2006, 74, 165419.

33. Saito, Y.; Ueta, T., Monte Carlo Studies of Equilibrium and Growth Shapes of a Crystal. Phys. Rev. A 1989, 40, 3408.

34. Baletto, F.; Mottet, C.; Ferrando, R., Molecular Dynamics Simulations of Surface Diffusion and Growth on Silver and Gold Clusters. Surf Sci. 2000, 446, 31-45.

35. Momma, K.; Izumi, F., Vesta: A Three-Dimensional Visualization System for Electronic and Structural Analysis. J. Appl. Crystallogr. 2008, 41, 653-658.

36. Momma, K.; Izumi, F., Vesta 3 for Three-Dimensional Visualization of Crystal, Volumetric and Morphology Data. J. Appl. Crystallogr. 2011, 44, 1272-1276.

37. Macrae, C. F.; Bruno, I. J.; Chisholm, J. A.; Edgington, P. R.; McCabe, P.; Pidcock, E.; Rodriguez-Monge, L.; Taylor, R.; Streek, J. v.; Wood, P. A., Mercury CSD 2.0-New Features for the Visualization and Investigation of Crystal Structures. J. Appl. Crystallogr. 2008, 41, 466-470.

38. Macrae, C. F.; Edgington, P. R.; McCabe, P.; Pidcock, E.; Shields, G. P.; Taylor, R.; Towler, M.; Streek, J. v. d., Mercury: Visualization and Analysis of Crystal Structures. J. Appl. Crystallogr. 2006, 39, 453-457.

39. Bruno, I. J.; Cole, J. C.; Edgington, P. R.; Kessler, M.; Macrae, C. F.; McCabe, P.; Pearson, J.; Taylor, R., New Software for Searching the Cambridge Structural Database and Visualizing Crystal Structures. Acta Crystallogr. Sect. B: Struct. Sci. 2002, 58, 389-397. 40. Taylor, R.; Macrae, C. F., Rules Governing the Crystal Packing of Mono-and Dialcohols. Acta Crystallogr. Sect. B: Struct. Sci. 2001, 57, 815-827.

41. Kaminsky, W., WinXMorph: A Computer Program to Draw Crystal Morphology, Growth Sectors and Cross Sections with Export Files in VRML V2.0 Utf8-Virtual Reality Format. J. Appl. Crystallogr. 2005, 38, 566-567.

42. Kaminsky, W., From CIF to Virtual Morphology Using the WinXMorph Program. $J$. Appl. Crystallogr. 2007, 40, 382-385.

43. Hiller, H., Crystallography and Cohomology of Groups. Amer. Math. Monthly 1986, 93, 765-779.

44. Wood, E. A., Vocabulary of Surface Crystallography. J. Appl. Phys. 1964, 35, 13061312.

45. Barmparis, G. D.; Lodziana, Z.; Lopez, N.; Remediakis, I. N., Nanoparticle Shapes by Using Wulff Constructions and First-Principles Calculations. Beilstein J. Nanotech. 2015, 6, 361.

46. Bennema, P.; Meekes, H.; Boerrigter, S.; Cuppen, H.; Deij, M.; Van Eupen, J.; Verwer, P.; Vlieg, E., Crystal Growth and Morphology: New Developments in an Integrated Hartman- Perdok Connected Net Roughening Transition Theory, Supported by Computer Simulations. Cryst. Growth Des. 2004, 4, 905-913. 
47. Fleming, S. D.; Rohl, A. L.; Parker, S. C.; Parkinson, G. M., Atomistic Modeling of Gibbsite: Cation Incorporation. J. Phys. Chem. B 2001, 105, 5099-5105.

48. Zou, Y.-C.; Chen, Z.-G.; Kong, F.; Zhang, E.; Drennan, J.; Cho, K.; Xiu, F.; Zou, J., Surface-Energy Engineered Bi-Doped Snte Nanoribbons with Weak Antilocalization Effect and Linear Magnetoresistance. Nanoscale 2016, 8, 19383-19389.

49. Tran, R.; Xu, Z.; Radhakrishnan, B.; Winston, D.; Sun, W.; Persson, K. A.; Ong, S. P., Surface Energies of Elemental Crystals. Sci. Data 2016, 3, 160080.

50. Donnay, J. D. H.; Harker, D., A New Law of Crystal Morphology Extending the Law of Bravais. Am. Mineral 1937, 22, 446-467.

51. Hartman, P.; Perdok, W., On the Relations between Structure and Morphology of Crystals. I. Acta Crystallogr. 1955, 8, 49-52.

52. Hartman, P.; Perdok, W., On the Relations between Structure and Morphology of Crystals. II. Acta Crystallogr. 1955, 8, 521-524.

53. Hartman, P.; Perdok, W., On the Relations between Structure and Morphology of Crystals. III. Acta Crystallogr. 1955, 8, 525-529.

54. Rijpkema, J.; Knops, H.; Bennema, P.; Van der Eerden, J., Determination of the Ising Critical Temperature of F Slices with an Application to Garnet. J. Cryst. Growth 1983, 61, 295-306.

55. Barber, C. B.; Dobkin, D. P.; Huhdanpaa, H., The Quickhull Algorithm for Convex Hulls. ACM Trans. Math. Softw. 1996, 22, 469-483.

56. Cordero, B.; Gómez, V.; Platero-Prats, A. E.; Revés, M.; Echeverría, J.; Cremades, E.; Barragán, F.; Alvarez, S., Covalent Radii Revisited. Dalton T. 2008, 2832-2838.

57. Santos-Carballal, D.; Roldan, A.; Grau-Crespo, R.; de Leeuw, N. H., A DFT Study of the Structures, Stabilities and Redox Behaviour of the Major Surfaces of Magnetite Fe3O4. Phys. Chem. Chem. Phys. 2014, 16, 21082-21097.

58. Ramamoorthy, M.; Vanderbilt, D.; King-Smith, R., First-Principles Calculations of the Energetics of Stoichiometric Tio 2 Surfaces. Phys. Rev. B 1994, 49, 16721.

59. Wang, D.; Xin, H. L.; Hovden, R.; Wang, H.; Yu, Y.; Muller, D. A.; DiSalvo, F. J.; Abruña, H. D., Structurally Ordered Intermetallic Platinum-Cobalt Core-Shell Nanoparticles with Enhanced Activity and Stability as Oxygen Reduction Electrocatalysts. Nat. Mater.

2013, 12, 81-87.

60. Su, D.; Dou, S.; Wang, G., Gold Nanocrystals with Variable Index Facets as Highly Effective Cathode Catalysts for Lithium-Oxygen Batteries. NPG Asia Mater. 2015, 7, e155.

61. Hanson, R. M., Jmol-a Paradigm Shift in Crystallographic Visualization. J. Appl. Crystallogr. 2010, 43, 1250-1260.

62. Hanson, R. M.; Prilusky, J.; Renjian, Z.; Nakane, T.; Sussman, J. L., Jsmol and the Next-Generation Web-Based Representation of 3D Molecular Structure as Applied to Proteopedia. Isr. J. Chem. 2013, 53, 207-216.

63. Merkys, A.; Vaitkus, A.; Butkus, J.; Okulič-Kazarinas, M.; Kairys, V.; Gražulis, S., COD::CIF::Parser: An Error-Correcting CIF Parser for the Perl Language. J. Appl.

Crystallogr. 2016, 49, 292-301.

64. Gražulis, S.; Merkys, A.; Vaitkus, A.; Okulič-Kazarinas, M., Computing Stoichiometric Molecular Composition from Crystal Structures. J. Appl. Crystallogr. 2015, $48,85-91$.

65. Gražulis, S.; Daškevič, A.; Merkys, A.; Chateigner, D.; Lutterotti, L.; Quiros, M.; Serebryanaya, N. R.; Moeck, P.; Downs, R. T.; Le Bail, A., Crystallography Open Database (COD): An Open-Access Collection of Crystal Structures and Platform for World-Wide Collaboration. Nucleic Acids Res. 2011, 40, D420-D427. 
66. Gražulis, S.; Chateigner, D.; Downs, R. T.; Yokochi, A.; Quirós, M.; Lutterotti, L.; Manakova, E.; Butkus, J.; Moeck, P.; Le Bail, A., Crystallography Open Database-an OpenAccess Collection of Crystal Structures. J. Appl. Crystallogr. 2009, 42, 726-729.

67. Downs, R. T.; Hall-Wallace, M., The American Mineralogist Crystal Structure Database. Am. Mineral. 2003, 88, 247-250.

68. Gatta, G. D.; Kantor, I.; Ballaran, T. B.; Dubrovinsky, L.; McCammon, C., Effect of Non-Hydrostatic Conditions on the Elastic Behaviour of Magnetite: An in Situ Single-Crystal X-Ray Diffraction Study. Phys. Chem. Miner. 2007, 34, 627-635.

69. Matlab and Statistics Toolbox, R2015a; The MathWorks, Inc.: Natick, Massachusetts, United States, 2015. 
TOC GRAPHIC

Any Crystallographic

Nanoparticle crystal Unit Cell shape

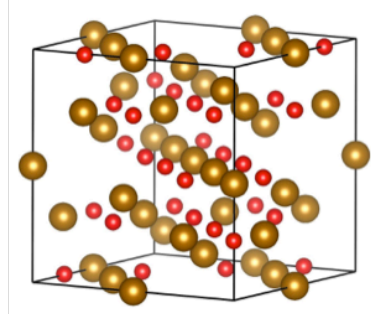

\section{NanoCrystal}

